\title{
Periodic Vortex Streets and Complex Monodromy
}

\author{
Adrian D. HEMERY ${ }^{\dagger}$ and Alexander P. VESELOV $\ddagger \S$ \\ $\dagger$ Charterhouse School, Godalming, Surrey, GU'7 2DX, UK \\ E-mail: adh@charterhouse.org.uk \\ $\ddagger$ Department of Mathematical Sciences, Loughborough University, \\ Loughborough, Leicestershire, LE11 3TU, UK \\ E-mail: A.P.Veselov@lboro.ac.uk \\ $\S$ Moscow State University, Russia
}

Received August 28, 2014, in final form December 10, 2014; Published online December 23, 2014 http://dx.doi.org/10.3842/SIGMA.2014.114

\begin{abstract}
The explicit constructions of periodic and doubly periodic vortex relative equilibria using the theory of monodromy-free Schrödinger operators are described. Several concrete examples with the qualitative analysis of the corresponding travelling vortex streets are given.
\end{abstract}

Key words: vortex; equilibria; monodromy; integrability

2010 Mathematics Subject Classification: 76B47; 34M05; 81R12

\section{Introduction}

The study of vortex dynamics is a classical subject going back to Helmholtz [35]. If we identify the plane with the set of complex numbers $\mathbb{C}$ then the dynamics of $N$ point vortices $z_{1}(t), \ldots, z_{N}(t)$ with circulations (or, vorticities) $\Gamma_{1}, \ldots, \Gamma_{N}$ is determined by the system

$$
\frac{d \bar{z}_{j}}{d t}=\frac{1}{2 \pi i} \sum_{k \neq j}^{N} \frac{\Gamma_{k}}{z_{j}-z_{k}}, \quad j=1, \ldots, N .
$$

In the periodic setting we have the equations

$$
\frac{d \bar{z}_{j}}{d t}=\frac{1}{2 \pi i} \sum_{k \neq j}^{N} \Gamma_{k} \cot \left(z_{j}-z_{k}\right), \quad j=1, \ldots, N,
$$

where we assume for simplicity that the period $L=\pi$ (see [4, 22] and the pioneering paper by Friedman and Polubarinova [14]).

In this paper we consider the periodic relative equilibria of the vortices described by the system

$$
\frac{1}{2 \pi i} \sum_{k \neq j}^{N} \Gamma_{k} \cot \left(z_{j}-z_{k}\right)-\bar{v}=0, \quad j=1, \ldots, N,
$$

where $v=\frac{d z_{j}}{d t}$ is the common constant velocity of the vortices. A classical example is given by the so-called von Kármán vortex street [20,36, 37], corresponding to the case $N=2, \Gamma_{1}+\Gamma_{2}=0$.

More recently the vortex dynamics in periodic domains was studied in [4, 22, 26, 31], but the dynamics in general is known to be non-integrable and even the description of all relative equilibria remains a largely open question. 
6

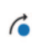

6

6

6

Figure 1. Classical von Kármán vortex street moving to the left.

One of the results of this paper is the following class of the explicit periodic relative vortex equilibria, most of which seem to be new.

Let $k=\left(k_{1}, \ldots, k_{n}\right), k_{1}<k_{2}<\cdots<k_{n}$ be a set of distinct natural numbers, and $\phi=$ $\left(\phi_{1}, \ldots, \phi_{n}\right), \phi_{j} \in \mathbb{C} / \pi \mathbb{Z}$ be a set of $n$ complex numbers considered modulo $\pi$. Let

$$
W_{k, \phi}(z)=W\left(\chi_{k_{1}, \phi_{1}}(z), \ldots, \chi_{k_{n}, \phi_{n}}(z)\right)
$$

be the Wronskian with $\chi_{k_{j}, \phi_{j}}(z)=\sin \left(k_{j} z+\phi_{j}\right)$ and

$$
W_{k, \phi, \kappa}(z)=W\left(\chi_{k_{1}, \phi_{1}}(z), \ldots, \chi_{k_{n}, \phi_{n}}(z), e^{i \kappa z}\right)
$$

where $\kappa \in \mathbb{C}$ is another complex parameter. Consider the combined configuration $\Sigma_{k, \phi, \kappa}$ of complex roots of $W_{k, \phi, \kappa}(z)=0$ and $W_{k, \phi}(z)=0$. Prescribe their circulations as follows: a zero $z_{i}$ of $W_{k, \phi, \kappa}(z)=0$ has the circulation $\Gamma_{i}=m_{i}$ equal to the multiplicity of $z_{i}$ while for a zero $z_{j}$ of $W_{k, \phi}(z)$ the circulation $\Gamma_{j}=-m_{j}$ is negative multiplicity of $z_{j}$ (for common zeros of $W_{k, \phi, \kappa}(z)$ and $W_{k, \phi}(z)$ the circulation is the difference of the corresponding multiplicities).

Theorem 1.1. The configuration $\Sigma_{k, \phi, \kappa}$ is a periodic relative vortex equilibrium moving with constant velocity $v=-\frac{\bar{\kappa}}{2 \pi}$ for any non-critical $\kappa \notin\left\{k_{1}, \ldots, k_{n}\right\}$. In the frame moving with the vortices the complex potential of the flow is

$$
W=\frac{1}{2 \pi i} \log \psi(\kappa, z),
$$

where

$$
\psi(\kappa, z)=\frac{W_{k, \phi, \kappa}(z)}{W_{k, \phi}(z)}
$$

is a trigonometric Baker-Akhiezer function for the corresponding monodromy-free Schrödinger operator

$$
L=-D^{2}+\sum \frac{m_{j}\left(m_{j}-1\right)}{\sin ^{2}\left(z-z_{j}\right)}, \quad D=\frac{d}{d z} .
$$

For critical value $\kappa=k_{j}$ we have an equilibrium vortex configuration with complex potential $W=\frac{1}{2 \pi i} \log \psi_{j}(z)$,

$$
\psi_{j}=\frac{W_{k^{(j)}, \phi^{(j)}}(z)}{W_{k, \phi}(z)}
$$

where $k^{(j)}, \phi^{(j)}$ are the sets $k, \phi$ without $k_{j}$ and $\phi_{j}$ respectively.

The proof (see Section 3 below) is based on a simple observation that the conditions of relative equilibrium coincide with the Stieltjes relations [33] and thus hold for all periodic trigonometric monodromy-free operators of the form (1.4). Such operators play an important role in the theory of Huygens principle as it was shown by Berest and Loutsenko [7]. They were classified in [6, 8] (see Theorem 4.3 in [8]) and all turned out to be iterated Darboux transformations applied to 
trivial potential $u=0$. For rational potentials a similar observation was known already for quite a while [5] (see also [3] and references therein), but in the periodic setting we have not seen this in the literature although it looks quite natural.

Von Kármán vortex streets correspond to the simplest case $n=1$. Indeed, let us consider for simplicity $k_{1}=1, \phi_{1}=0$, then

$$
\psi(\kappa, z)=(i \kappa-\cot z) e^{i \kappa z} .
$$

We have one zero and one pole modulo $\pi$ : the pole is $z=0$ and the zero is the solution of $\cot z=i \kappa$, which is equivalent to

$$
e^{2 i z}=\frac{\kappa+1}{\kappa-1}
$$

if $\kappa \neq 1$. Assuming that $\kappa$ is real and positive, we have 2 cases: $\kappa>1$ (fast), $\kappa<1$ (slow) when

$$
z=\frac{1}{2 i} \log \frac{\kappa+1}{\kappa-1} \quad \text { and } \quad z=\frac{1}{2 i} \log \frac{\kappa+1}{1-\kappa}+\frac{\pi}{2}
$$

respectively. In the slow case we have the von Kármán street shown in Fig. 1, the fast case is shown in Fig. 2.

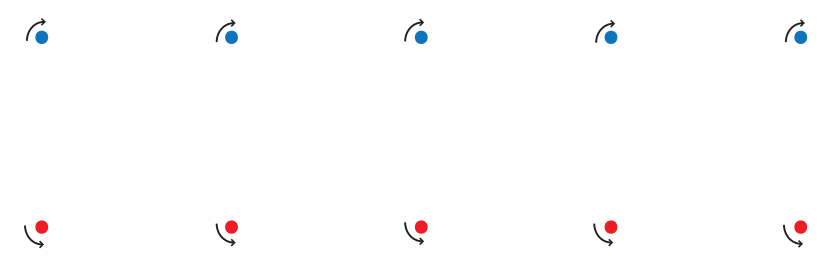

Figure 2. Fast von Kármán vortex street, known to be unstable.

The complex potential of the flow in the moving frame is $W=\frac{1}{2 \pi i} \log \psi(\kappa, z)$, the instantaneous complex potential of the flow in the fixed frame is

$$
W=\frac{1}{2 \pi i} \log \psi(\kappa, z) e^{-i \kappa z}=\frac{1}{2 \pi i} \log (i \kappa-\cot z) .
$$

For the critical value $\kappa=1$ the zero of $\psi$ goes to infinity: $\psi=\frac{1}{\sin z}$ and we have trivial vortex equilibrium, consisting of points $l \pi, l \in \mathbb{Z}$ with circulations -1 .

Two examples in the case $n=2$ are shown in Fig. 3 , the details and more examples with some qualitative analysis are presented in Section 6. All the pictures in the paper were produced using Mathematica. The colour of the points indicates the sign of the circulations (which are generically \pm 1$)$ : red means positive, blue - negative circulations. The axes on all figures are $x$ and $y$, such that $z=x+i y$.

Our approach based on the ideas of [33] is very general and can be applied to all monodromyfree operators. In particular, we apply it to the classical Whittaker-Hill operator and its Darboux transformations [17] to construct periodic equilibria in the presence of background flow with complex potential $W=A \cos 2 z$.

In the doubly periodic case we have more general result, when only one meromorphic solution is required. Let $\sigma(z)$ and $\wp(z)$ be classical Weierstrass elliptic functions [39].

Theorem 1.2. Let

$$
\psi(z)=\prod_{i=1}^{N} \sigma^{m_{i}}\left(z-z_{i}\right) e^{B z}
$$



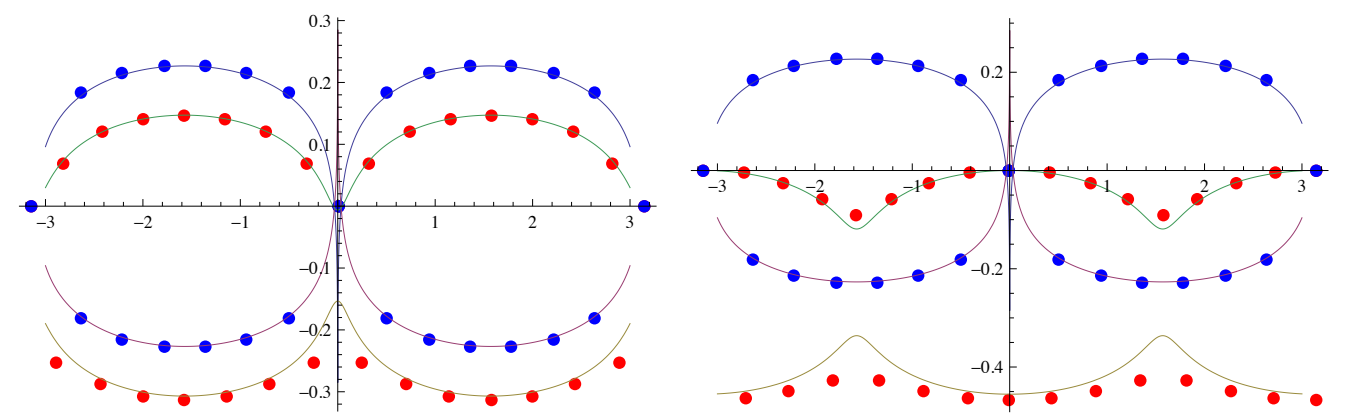

Figure 3. Vortex streets $\Sigma_{k, 0, \kappa}$ with $k=(7,8), \kappa=4$ (left) and $\kappa=7.4$ (right) with the graphs of the asymptotic curves (6.3), (6.4).

with $m_{i} \in \mathbb{Z}, m_{1}+\cdots+m_{N}=0$ and $B \in \mathbb{C}$ be a solution of the Schrödinger equation

$$
-\psi^{\prime \prime}+\left(\sum_{i=1}^{N} m_{i}\left(m_{i}-1\right) \wp\left(z-z_{i}\right)\right) \psi=E \psi .
$$

Then $W(z)=\frac{1}{2 \pi i} \log \psi(z)$ is the complex potential of a doubly periodic relative vortex equilibrium in the moving frame.

Conversely, let the set $z_{1}, \ldots, z_{N}$ with integer circulations $m_{1}, \ldots, m_{N}$ with zero sum be a doubly periodic relative vortex equilibrium. Then the function (1.5) with suitable constant $B$ is a solution of equation (1.6) for some energy $E$.

The classical theory of Lamé equation going back to Hermite [39] and modern theory of elliptic solitons $[2,9,19,24,28,29,32,34]$, which can be defined as the theory of monodromyfree operators of the form (1.6) (or Picard potentials in terminology of [15]), provides many examples of such solutions and thus new doubly periodic relative equilibria. In particular, the logarithm of the corresponding elliptic Baker-Akhiezer function is a complex potential for a relative doubly periodic vortex equilibrium in the moving frame. We should mention though that in contrast to trigonometric case an effective description of all elliptic finite-gap (or algebrogeometric) operators still remains an open problem, which was first emphasized by S.P. Novikov as part of the effectivisation programme in finite-gap theory.

Note that in all the examples we produce the circulations are integers, which might be useful for applications to liquid helium, where circulations are known to be quantized $[13,30]$.

\section{Monodromy-free Schrödinger operators and Stieltjes relations}

Consider the Schrödinger operator

$$
L=-D^{2}+u(z)
$$

in the complex domain $z \in \mathbb{C}$ with meromorphic potential $u(z)$ having poles only of second order. The operator $L$ is called monodromy-free if the corresponding Schrödinger equation

$$
-\varphi^{\prime \prime}+u(z) \varphi=E \varphi
$$

has all solutions meromorphic for all values of $E$.

Near a pole (which can be assumed for simplicity to be $z=0$ ) the potential can be represented

as Laurent series $u=\sum_{i=-2}^{\infty} c_{i} z^{i}$. Following the classical Frobenius approach one can look for the 
solutions of the form

$$
\varphi=z^{-\mu}\left(1+\sum_{i=1}^{\infty} \xi_{i} z^{i}\right) .
$$

The corresponding $\mu$ must satisfy the characteristic equation $\mu(\mu+1)=c_{-2}$, which means that the equation (2.1) has a meromorphic solution only if the coefficient $c_{-2}$ at any pole has a very special form:

$$
c_{-2}=m(m+1), \quad m \in \mathbf{Z}_{+} .
$$

This condition is in fact not sufficient: the corresponding solution $\varphi$ may have a logarithmic term. The following important lemma due to Duistermaat and Grünbaum [10] gives the conditions when this does not happen.

Lemma 2.1 ([10]). The logarithmic terms are absent for all $\lambda$ if and only if in addition to (2.2) all the first $m+1$ odd coefficients at the Laurent expansion of the potential are vanishing:

$$
c_{2 k-1}=0, \quad k=0,1, \ldots, m
$$

Let $\psi(z)$ be a solution of the corresponding Schrödinger equation

$$
\left(-D^{2}+u(z)\right) \psi(z)=\lambda \psi(z)
$$

and $f(z)=D \log \psi(z)$. Then the potential $u(z)$ can be expressed as

$$
u(z)=f^{\prime}+f^{2}+\lambda .
$$

Proposition 2.2 ([33]). Let $f$ be a meromorphic function having the poles of the first order with integer residues. The Schrödinger operator $L$ with the potential $u=f^{\prime}+f^{2}+$ const is monodromy-free if and only if at any pole $z_{0}$ with $\operatorname{Res}_{z=z_{0}} f=m$ the following generalised Stieltjes relations are satisfied:

$$
\operatorname{Res}_{z=z_{0}} f^{2}=\operatorname{Res}_{z=z_{0}} f^{4}=\cdots=\operatorname{Res}_{z=z_{0}} f^{2|m|}=0 .
$$

The proof is simple: substituting

$$
f=\frac{ \pm m}{z-z_{0}}+\sum_{k=0} \alpha_{k}\left(z-z_{0}\right)^{k}
$$

with $m \in \mathbb{Z}_{+}$into $u=f^{\prime}+f^{2}$ that $c_{-2}=m(m \pm 1)$ we can check that the trivial monodromy conditions $c_{2 k-1}=0, k=0,1, \ldots, m-1$ are equivalent to the vanishing of the coefficients $\alpha_{2 k}=0, k=0,1, \ldots, m-1$. The last relation $c_{2 m-1}=0$ is then fulfilled automatically, see [33].

In particular, we always have the original Stieltjes relation: at every pole $z_{i}$ of $f$

$$
\operatorname{Res}_{z=z_{i}} f^{2}=0
$$

which Stieltjes used to give electrostatic interpretation of the zeroes of some classical polynomials [25]. We are going to use the same idea to produce some new relative vortex equilibria. 


\section{Trigonometric monodromy-free operators and periodic vortex streets}

As we have already mentioned all $\pi$-periodic trigonometric monodromy-free operators of the form (1.4) are known $[6,8]$ to be the result of several Darboux transformations applied to $L_{0}=-D^{2}$.

The corresponding potentials have the form

$$
u(z)=-2 D^{2} \log W\left(\chi_{k_{1}, \phi_{1}}(z), \ldots, \chi_{k_{n}, \phi_{n}}(z)\right),
$$

where $k_{1}<k_{2}<\cdots<k_{n}$ are distinct natural numbers, $\phi_{i} \in \mathbb{C} / \pi \mathbb{Z}$ are arbitrary complex numbers modulo $\pi$,

$$
\chi_{l, \phi}(z)=\sin (l z+\phi), \quad l \in \mathbb{N} .
$$

The Schrödinger equation

$$
\left(-D^{2}+u(z)\right) \psi=\kappa^{2} \psi
$$

with $u(z)$ given by (3.1) has solution

$$
\psi(\kappa, z)=\frac{W_{n}(\kappa, z)}{W_{n}(z)},
$$

where $W_{n}(z)=W_{k, \phi}(z), W_{n}(\kappa, z)=W_{k, \phi, \kappa}(z)$ are the Wronskians (1.2), (1.3). Note that $W_{n}(\kappa, z)=P_{n}(\kappa, z) e^{i \kappa z}$, where $P_{n}(\kappa, z)=W_{n}(z) i^{n} \kappa^{n}+\sum_{j=0}^{n-1} A_{j}(z) \kappa^{j}$ is a polynomial in $\kappa$ with coefficients being trigonometric polynomials in $z$, so the ratio $\psi(\kappa, z)=W_{n}(\kappa, z) / W_{n}(z)$ is (a version of) the corresponding trigonometric Baker-Akhiezer function [18].

Let

$$
W_{n}(z)=C \prod_{i=1}^{M} \sin ^{m_{i}}\left(z-a_{i}\right), \quad W_{n}(\kappa, z)=C^{\prime} \prod_{i=1}^{N} \sin ^{n_{i}}\left(z-b_{i}\right) e^{i \kappa z}
$$

with some constants $C$ and $C^{\prime}$ be the corresponding factorisations with possible multiplicities. Then the log-derivative $f=D \log \psi(\kappa, z)=D \log W_{n}(\kappa, z)-D \log W_{n}(z)$ has the form

$$
f=\sum_{i=1}^{M} m_{i} \cot \left(z-b_{i}\right)-\sum_{j=1}^{N} n_{j} \cot \left(z-a_{j}\right)+i \kappa=\sum_{i=1}^{M+N} \Gamma_{i} \cot \left(z-z_{i}\right)+i \kappa
$$

where $z_{1}, \ldots, z_{M+N}=a_{1}, \ldots, a_{M}, b_{1}, \ldots, b_{N}$ and $\Gamma_{i}=m_{i}$ for $i=1, \ldots, M$ and $\Gamma_{M+j}=-n_{j}$ for $j=1, \ldots, N$. It may happen that $a_{i}=b_{j}$, so we have a cancelation with $\Gamma=m_{i}-n_{j}$ in that case.

We claim that the set of vortices with position at $z_{1}, \ldots, z_{M+N}$ with the corresponding circulations $\Gamma_{1}, \ldots, \Gamma_{M+N}$ described above is a periodic relative vortex equilibrium configuration moving with velocity $v=\bar{\kappa} / 2 \pi$. Indeed, by Stieltjes relations

$$
\operatorname{Res}_{z=z_{j}} f^{2}=2 \Gamma_{j} \sum_{k \neq j} \Gamma_{k} \cot \left(z_{j}-z_{k}\right)+2 i \Gamma_{j} \kappa=0
$$

for all $j=1, \ldots, M+N$. Comparing with (1.1) we see that

$$
\frac{d \bar{z}_{j}}{d t}=\frac{1}{2 \pi i} \sum_{k \neq j} \Gamma_{k} \cot \left(z_{j}-z_{k}\right)=-\frac{\kappa}{2 \pi}
$$

so we have the vortex configuration moving with constant speed $v=-\bar{\kappa} / 2 \pi$. 
Note that in the frame moving with the vortices the corresponding flow can be written as

$$
\frac{d \bar{z}}{d t}=\frac{1}{2 \pi i}\left(\sum_{i=1}^{M} m_{i} \cot \left(z-b_{i}\right)-\sum_{j=1}^{N} n_{j} \cot \left(z-a_{j}\right)+i \kappa\right)=\frac{d}{d z} \frac{1}{2 \pi i} \log \psi,
$$

so by definition $W=\frac{1}{2 \pi i} \log \psi(\kappa, z)$ is the complex potential of the flow [1,21] $]^{1}$. The instantaneous complex potential of the flow in the fixed frame is

$$
W_{*}=W-\frac{\kappa}{2 \pi} z=\frac{1}{2 \pi i} \log \left(\psi e^{-i \kappa z}\right)=\frac{1}{2 \pi i} \log \frac{P_{n}(\kappa, z)}{W_{n}(z)} .
$$

At the critical level $\kappa=k_{j}$ the Wronskian $W_{k, \phi, \kappa}(z)$ reduces to

$$
W_{k, \phi, k_{j}}(z)=W_{k^{(j)}, \phi^{(j)}}(z),
$$

so we have

$$
\psi=\frac{W_{k^{(j)}, \phi^{(j)}}(z)}{W_{k, \phi}(z)},
$$

where $k^{(j)}=\left(k_{1}, \ldots, \hat{k}_{j}, \ldots, k_{n}\right)$, where $\hat{k}_{j}$ means that $k_{j}$ is omitted from the list, and similarly for $\phi^{(j)}$. Some of the zeros disappear at infinity and the relative equilibriium becomes a genuine one, in agreement with the general claim by Montaldi, Solière and Tokieda [22] that if the sum of the vorticities is not zero the only relative equilibria are the usual equilibria. This completes the proof of Theorem 1.1.

We will discuss many examples of corresponding relative vortex equilibria in Section 6 . Here we will mention only new collinear vortex equilibria (when all the vortices are on a real line), related to Baker-Akhiezer configurations found by M. Feigin and D. Johnston [11].

The corresponding vortex equilibrium $\Sigma(n, m, l)$ depends on integer parameters $n>m \geq 1$ and even $l>0$. It corresponds to

$$
k=(1,2, \ldots, n-m, n-m+2, n-m+4, \ldots, n+m-2, n+m+l)
$$

(or, more explicitly $k_{j}=j$ for $j=1, \ldots, n-m, k_{n-m+j}=n-m+2 j$ for $j=1, \ldots, m-1$ and $\left.k_{n}=n+m+l\right)$ and $\phi=0$. One can show that in this case

$$
\begin{aligned}
& W_{k^{(n)}, \phi^{(n)}}(z)=C_{1} \cos ^{\frac{m(m-1)}{2}} z \sin ^{\frac{n(n-1)}{2}} z, \\
& W_{k, \phi}(z)=C_{2} \cos ^{\frac{m(m+1)}{2}} z \sin ^{\frac{n(n+1)}{2}} z \prod_{j=1}^{l} \sin \left(z-z_{j}\right),
\end{aligned}
$$

where $z_{1}, \ldots, z_{l} \in(0, \pi)$ are certain simple real zeros located symmetrically with respect to $\pi / 2$ (see [11]). This means that the function $W=\frac{1}{2 \pi i} \log \psi(z)$ with

$$
\psi(z)=W_{k, \phi}(z) / W_{k^{(n)}, \phi^{(n)}}(z)=C \cos ^{m} z \sin ^{n} z \prod_{j=1}^{l} \sin \left(z-z_{j}\right)
$$

is the complex potential of the collinear vortex equilibrium configuration $\Sigma(n, m, l)$ consisting of $z=0$ with circulation $n, z=\pi / 2$ with circulation $m$ and $z=z_{1}, \ldots, z_{l}$ with circulation 1 .

One can produce more equilibriums by changing $z \rightarrow q z$ and extend this configuration to a moving vortex street by considering $\psi(z)=W_{k, \phi, \kappa}(z) / W_{k, \phi}(z)$ with non-critical $\kappa$.

\footnotetext{
${ }^{1}$ There is a sign discrepancy in the definition of the complex potential of the flow between Milne-Thomson [21] and Acheson [1]. We follow Acheson here.
} 


\section{Whittaker-Hill equation and relative vortex equilibria in the background flow}

Since the Darboux transformation preserves the class of monodromy-free operators one can use it in a more general situation to produce vortex equilibria in the presence of a background flow.

We demonstrate it here in the example of the classical Whittaker-Hill equation

$$
-\psi^{\prime \prime}-\left(4 \alpha s \cos 2 x+2 \alpha^{2} \cos 4 x\right) \psi=\lambda \psi \text {. }
$$

This equation is special because for natural values of parameter $s$ it has precisely $s$ elementary eigenfunctions of the form $\psi_{j}(z)=\varphi_{j}(z) e^{\alpha \cos 2 x}, j=1, \ldots, s$, where $\varphi_{j}(z)$ are some trigonometric polynomials [38]. For example, for $s=3$ we have

$$
\varphi_{1}=1+\frac{\sqrt{1+16 \alpha^{2}}-1}{4 \alpha} \cos 2 x, \quad \varphi_{2}=\sin 2 x, \quad \varphi_{3}=\frac{1-\sqrt{1+16 \alpha^{2}}}{4 \alpha}+\cos 2 x .
$$

Let $I=\left\{i_{1}, \ldots, i_{n}\right\}$ be a set of distinct natural numbers,

$$
W_{I}=W\left(\psi_{i_{1}}, \ldots, \psi_{i_{n}}\right)=W\left(\varphi_{i_{1}}, \ldots, \varphi_{i_{n}}\right) e^{n \alpha \cos 2 x}
$$

be the Wronskian of the corresponding eigenfunctions. Following [17] consider the Darboux transformation of the Whittaker-Hill operator with the potential

$$
\tilde{u}=-\left(4 \alpha s \cos 2 x+2 \alpha^{2} \cos 4 x\right)-2 D^{2} \log W_{I},
$$

and its eigenfunction $\psi_{J I}(z)=W_{J}(z) / W_{I}(z)$, where $J=\left\{i_{1}, \ldots, i_{n}, i_{n+1}\right\}$,

$$
W_{J}=W\left(\psi_{i_{1}}, \ldots, \psi_{i_{n}}, \psi_{i_{n+1}}\right)=W\left(\varphi_{i_{1}}, \ldots, \varphi_{i_{n}}, \varphi_{i_{n+1}}\right) e^{(n+1) \alpha \cos 2 x} .
$$

The corresponding log-derivative $f=D \log \psi_{J I}(z)$ has the form

$$
f=\sum_{i=1}^{M} m_{i} \cot \left(z-b_{i}\right)-\sum_{j=1}^{N} n_{j} \cot \left(z-a_{j}\right)-2 \alpha \sin 2 z=\sum_{i=1}^{M+N} \Gamma_{i} \cot \left(z-z_{i}\right)-2 \alpha \sin 2 z,
$$

where as before $a_{i}$ and $b_{j}$ are the zeros of the denominator $W_{I}$ and numerator $W_{J}$ with circulations being negative multiplicities and multiplicities respectively.

By Proposition 2.2 we have

$$
\operatorname{Res}_{z=z_{i}} f^{2}=2 \Gamma_{i} \sum_{j \neq i} \Gamma_{j} \cot \left(z_{i}-z_{j}\right)-4 \Gamma_{i} \alpha \sin 2 z_{i}=0,
$$

or,

$$
\sum_{j \neq i} \Gamma_{j} \cot \left(z_{i}-z_{j}\right)-2 \alpha \sin 2 z_{i}=0
$$

for all $i=1, \ldots, M+N$. These Stieltjes relations can be interpreted as the vortex equilibrium relations in a periodic background flow (see Fig. 4).

Theorem 4.1. The function $W=\frac{1}{2 \pi i} \log \psi_{J I}(z)=\frac{1}{2 \pi i}\left(\log W_{J}(z)-\log W_{I}(z)\right)$ is the complex potential of a vortex equilibrium in the background flow with complex potential $\frac{\alpha}{2 \pi i} \cos 2 z$.

Two examples of vortex equilibria in the case $s=5$ are shown in Fig. 5 . 


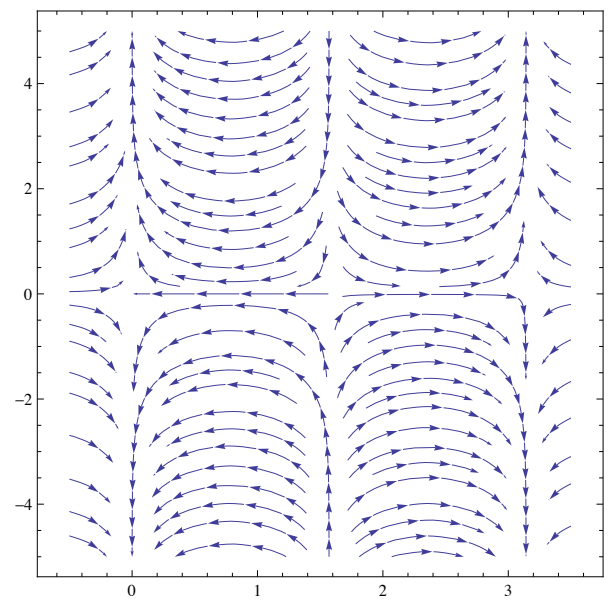

Figure 4. The background flow with complex potential $W=\cos 2 z$.

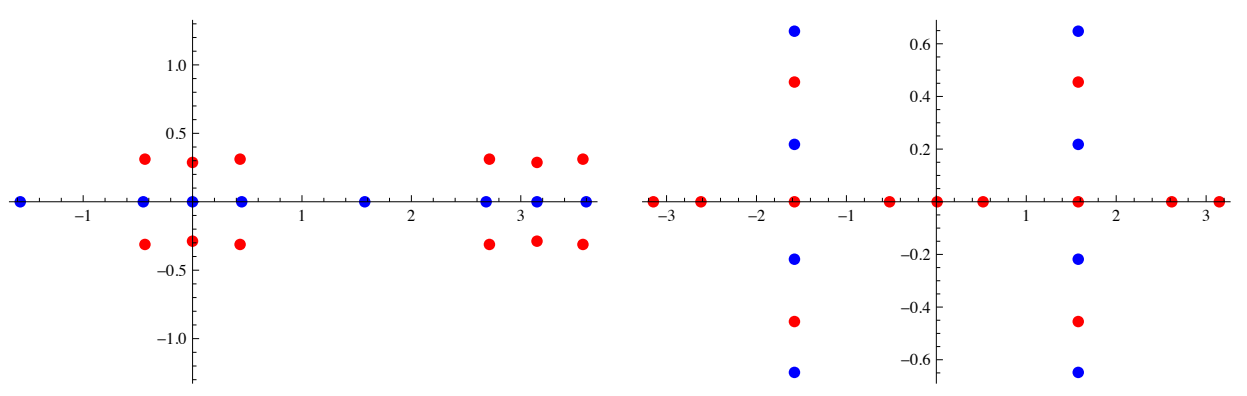

Figure 5. Vortex equilibria in the Whittaker-Hill case with $s=5, \alpha=1.5$ and $J=\{4,5\}, I=\{4\}$ (left), $J=\{1,5\}, I=\{1\}$ (right).

\section{Doubly-periodic vortex equilibria}

The classical Lamé operator has the form

$$
L=-D^{2}+s(s+1) \wp(z),
$$

where $\wp(z)$ is the Weierstrass elliptic function and $s$ is an integer. For the Lamé operator there are explicit formulae for the $2 s+1$ eigenfunctions, known as Lamé functions, going back to Hermite [39]. Replacing in the previous section $\psi_{i}(z)$ by the Lamé functions we have new doubly periodic vortex equilibria.

In fact, we have the following very general result. Let $\sigma(z), \zeta(z), \wp(z)$ be classical Weierstrass functions with periods $2 \omega_{1}, 2 \omega_{2}$, see Whittaker-Watson [39].

Suppose that

$$
\psi(z)=\prod_{i=1}^{N} \sigma^{m_{i}}\left(z-z_{i}\right) e^{B z}
$$

with integer $m_{i}$ with zero sum, is a solution of the Schrödinger equation

$$
-\psi^{\prime \prime}+u(z) \psi=E \psi
$$

with the potential

$$
u(z)=\sum_{i=1}^{N} m_{i}\left(m_{i}-1\right) \wp\left(z-z_{i}\right) .
$$


Then we claim that $W(z)=\frac{1}{2 \pi i} \log \psi(z)$ is the complex potential of a relative doubly periodic vortex equilibrium in the moving frame. Indeed, the function

$$
f=D \log \psi=\sum_{i=1}^{N} m_{i} \zeta\left(z-z_{i}\right)+B
$$

must satisfy the Riccati equation

$$
f^{\prime}+f^{2}=u(z)-E .
$$

Since the residues of the potential are zero, the same must be true for $f^{2}$, which implies the Stieltjes conditions

$$
\sum_{j \neq i}^{N} m_{j} \zeta\left(z_{i}-z_{j}\right)+B=0, \quad i=1, \ldots, N .
$$

Now recall the equations for the dynamics of the doubly periodic configuration of vortices. Let $z_{1}, \ldots, z_{N}$ with circulations $\Gamma_{1}, \ldots, \Gamma_{N}$ be part of such configuration within the parallelogram defined by the periods $2 \omega_{1}, 2 \omega_{2}$. Then their dynamics is described by the equations

$$
\frac{d \bar{z}_{k}}{d t}=\frac{1}{2 \pi i}\left(\sum_{j \neq k}^{N} \Gamma_{j} \zeta\left(z_{k}-z_{j}\right)+C\right), \quad i=1, \ldots, N
$$

where

$$
C=a \sum_{j=1}^{N} \Gamma_{j} z_{j}+b \sum_{j=1}^{N} \Gamma_{j} \bar{z}_{j}
$$

and $a, b$ are solutions of the linear system

$$
a \omega_{i}+b \bar{\omega}_{i}=\eta_{i}, \quad i=1,2,
$$

with the usual notation $\eta_{i}=\zeta\left(\omega_{i}\right)$ (see $[27,30]$ ). If $z_{1}, \ldots, z_{N}$ are the zeros of $\psi$ and $\Gamma_{i}=m_{i}$, then due to Stieltjes relations (5.1) we have

$$
\frac{d \bar{z}_{k}}{d t}=\frac{1}{2 \pi i}(C-B)=A,
$$

which shows that the configuration of zeros of $\psi$ is indeed a relative vortex equilibrium moving with velocity $v=\bar{A}$. The complex potential of the flow in the moving frame is $W=\frac{1}{2 \pi i} \log \psi(z)$, the instantaneous one in the fixed frame is

$$
W_{*}=W+A z=\frac{1}{2 \pi i} \log \left(\psi e^{(C-B) z}\right) .
$$

Conversely, assume that we have a doubly periodic relative vortex equilibrium $z_{1}, \ldots, z_{N}$ with integer circulations $\Gamma_{i}=m_{i}$, so that

$$
\frac{d \bar{z}_{k}}{d t}=\frac{1}{2 \pi i}\left(\sum_{j \neq k}^{N} \Gamma_{j} \zeta\left(z_{k}-z_{j}\right)+C\right)=A
$$

then we have Stieltjes relations (5.1) with $B=C-2 \pi i A$, which guarantee the Riccati relation (since the functions are elliptic) and hence the Schrödinger equation of the required form. This proves Theorem 1.2. 
Of course, in that form the claim becomes almost a tautology since both conditions are equivalent to the Stieltjes relations (5.1), which it is not clear how to solve. Fortunately we have several concrete examples coming from the theory of elliptic solitons [2, 9, 15, 19, 24, 28, 29, 32], which will be discussed elsewhere.

Here we only mention classical Hermite's solution of the Lamé equation $L \psi=E \psi$

$$
\psi=\prod_{r=1}^{s} \frac{\sigma\left(a_{r}-z\right)}{\sigma(z) \sigma\left(a_{r}\right)} e^{B z}, \quad B=\sum_{r=1}^{s} \zeta\left(a_{r}\right),
$$

which gives doubly periodic vortex streets with $z=0$ of circulation $-s$ and $z_{r}=a_{r}, r=1, \ldots, s$ of circulation 1, depending on an arbitrary parameter $E$ (see the details in Whittaker and Watson [39, Section 23.7]).

\section{Examples: pictures and analysis}

We restrict ourselves with the analysis of the vortex configurations given by Theorem 1.1. As we will see in spite of the simple explicit formulae there are many natural questions to answer already in this case.

As we have seen above, in the simplest case $n=1$ we have the original von Kármán vortex streets $[20,36,37]$.

\subsection{Case $n=2$ with $k_{1}=1, k_{2}=2$}

An example of the corresponding vortex street is shown at Fig. 6.

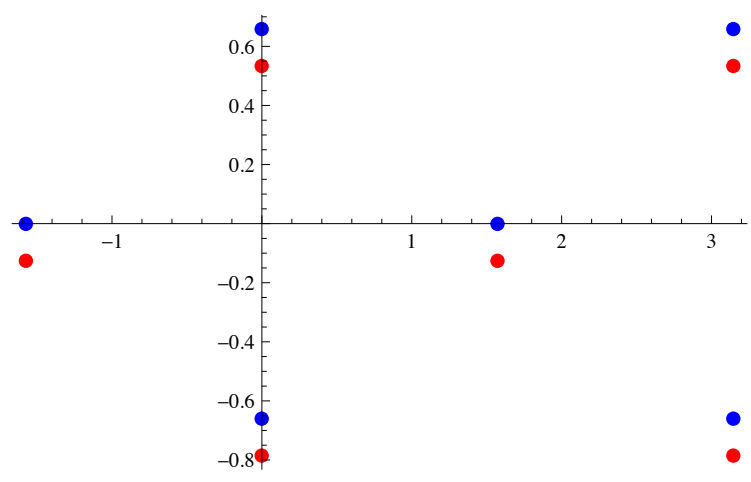

Figure 6. Configuration $\Sigma_{k, \phi, \kappa}$ with $k=(1,2), \phi=(0, \pi / 2)$ and $\kappa=8$. The circulations of the red and blue vortices are $\Gamma=1$ and $\Gamma=-1$ respectively.

Let us assume that $\phi_{1}=\phi_{2}=0$. As we will see in that case because of the cancelation we have only three vortices per period: one with circulation -2 (located at 0 ) and two with circulation +1 .

Indeed in this case $W(\sin z, \sin 2 z)=-2 \sin ^{3} z$ and

$$
W\left(\sin z, \sin 2 z, e^{i \kappa z}\right)=-\sin z e^{i \kappa z}\left(\left(2+\kappa^{2}\right) \cos 2 z-3 i \kappa \sin 2 z+\left(4-\kappa^{2}\right)\right),
$$

so we have the cancelation of $\sin z$ and

$$
\psi=\frac{W\left(\sin z, \sin 2 z, e^{i \kappa z}\right)}{W(\sin z, \sin 2 z)}=\frac{\left(2+\kappa^{2}\right) \cos 2 z-3 i \kappa \sin 2 z+\left(4-\kappa^{2}\right)}{2 \sin ^{2} z} e^{i \kappa z} .
$$

The equation

$$
\left(2+\kappa^{2}\right) \cos 2 z-3 i \kappa \sin 2 z+\left(4-\kappa^{2}\right)=0
$$


can be rewritten as

$$
(\kappa-1)(\kappa-2) e^{2 i z}+(\kappa+1)(\kappa+2) e^{-2 i z}-2(\kappa+2)(\kappa-2)=0,
$$

which is a quadratic in $X=e^{2 i z}$ with roots

$$
X_{1,2}=\frac{\left(\kappa^{2}-4\right) \pm \sqrt{3\left(4-\kappa^{2}\right)}}{(\kappa-1)(\kappa-2)} .
$$

So, we have a relative equilibrium with the vortices at the following locations (and $\pi$-periodically)

$$
\begin{aligned}
& z_{0}=0 \quad \text { with } \quad \Gamma=-2, \\
& z_{1,2}=\frac{1}{2 i} \log \left(\frac{\left(\kappa^{2}-4\right) \pm \sqrt{3\left(4-\kappa^{2}\right)}}{(\kappa-1)(\kappa-2)}\right) \quad \text { with } \quad \Gamma=1
\end{aligned}
$$

moving with the velocity $v=-\bar{\kappa} / 2 \pi$ for non-critical $\kappa \neq 1,2$.

When $\kappa=0$ we have $X_{1,2}=-2 \pm \sqrt{3}$ and an equilibrium configuration shown on the very left of Fig. 7 with red vortices $z_{1,2}=\pi / 2 \pm \frac{i}{2} \log (2+\sqrt{3})$. Figs. 7-10 show what happens when the parameter $\kappa$ grows.
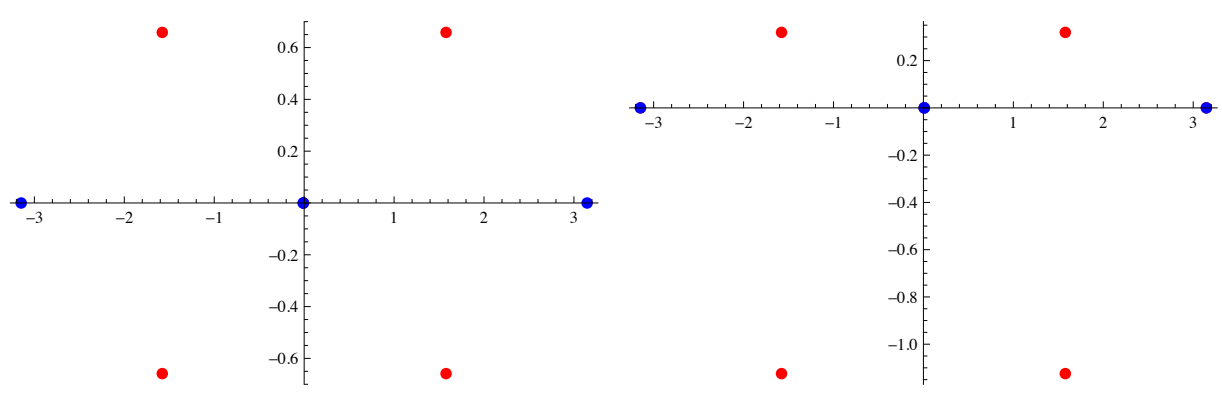

Figure 7. $\Sigma_{k, 0, \kappa}$ with $k=(1,2)$ and $\kappa=0$ (equilibrium, L), $\kappa=0.5(\mathrm{R})$. Circulation of the fixed vortex at 0 is -2 , while the other vortices have circulation 1 .
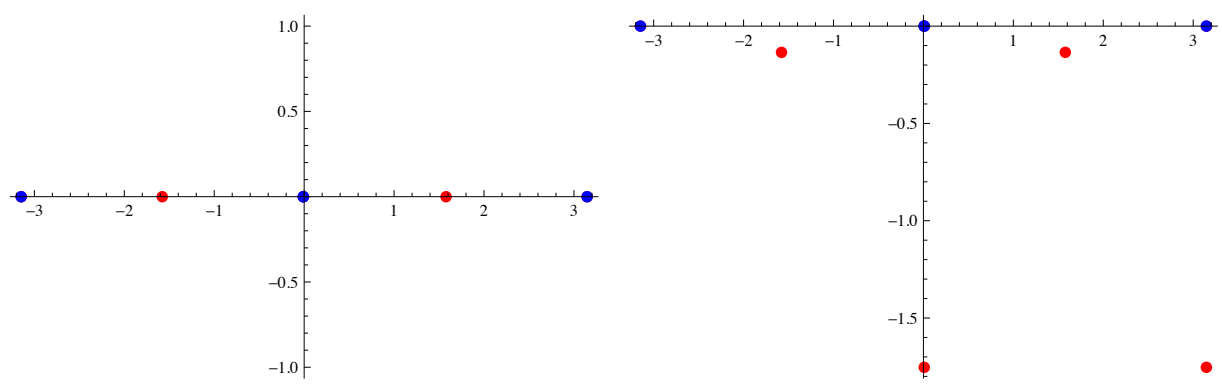

Figure 8. $\Sigma_{k, 0, \kappa}$ with $k=(1,2)$ and $\kappa=1$ (critical, L), $\kappa=1.2(\mathrm{R})$.

Let us look at what happens near the critical values $\kappa=1$ and $\kappa=2$. Let $\kappa=1+\epsilon$ with small $\epsilon$, then

$$
z_{1,2} \approx \frac{1}{2 i} \log \left(\frac{(-3+2 \epsilon) \pm(3-\epsilon)}{-\epsilon}\right)
$$

so the limiting value is $z_{1}=\frac{1}{2 i} \log (-1)=\frac{1}{2 i} \log e^{i \pi}=\frac{\pi}{2}$ while $z_{2} \approx \frac{1}{2 i} \log (6 / \epsilon) \rightarrow-i \infty$ goes to infinity. At the critical value $\kappa=1$ we have a genuine equilibrium shown at Fig. 8 with 0 and $\pi / 2$ having circulations -2 and 1 respectively corresponding to $\psi=\sin 2 z / \sin ^{3} z=2 \cos z / \sin ^{2} z$. 


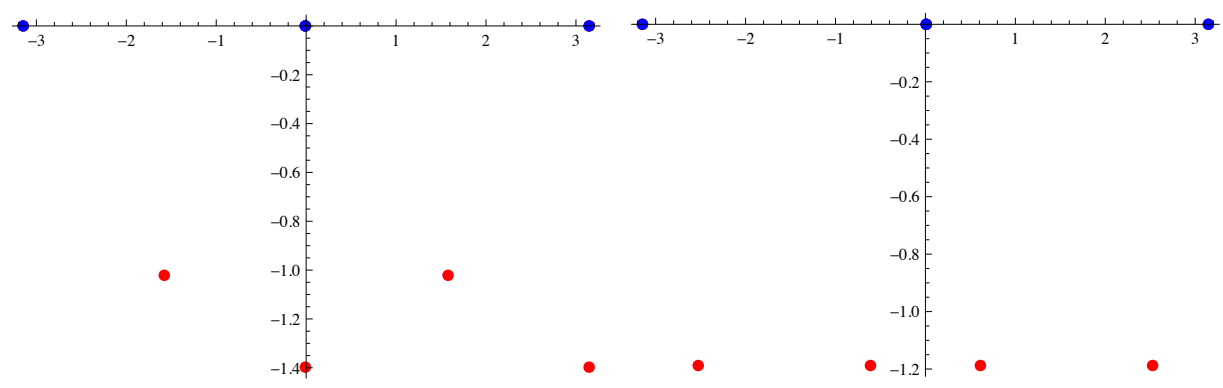

Figure 9. $\Sigma_{k, 0, \kappa}$ with $k=(1,2)$ and $\kappa=1.9(\mathrm{~L}), \kappa=2.1(\mathrm{R})$.

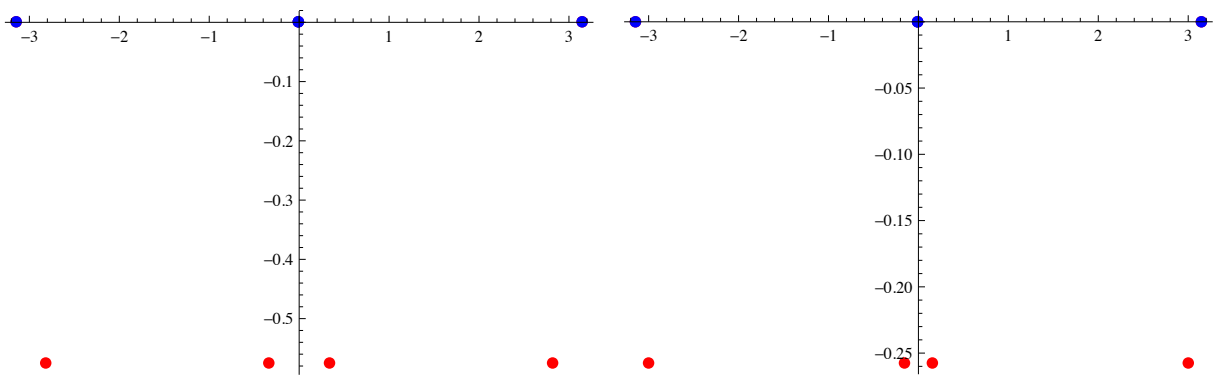

Figure 10. $\Sigma_{k, 0, \kappa}$ with $k=(1,2)$ and $\kappa=3(\mathrm{~L}), \kappa=6(\mathrm{R})$

Similarly, setting $\kappa=2+\epsilon, \epsilon>0$ small

$$
z_{1,2} \approx \frac{1}{2 i} \log \left(\frac{4 \epsilon \pm(-12 \epsilon)^{1 / 2}}{\epsilon}\right) \approx \frac{1}{2 i} \log \left( \pm 2 i(3 / \epsilon)^{1 / 2}\right),
$$

so in the limit $\epsilon \rightarrow 0 z_{1,2}$ go to $-i \infty$ with the real parts approaching $\pm \pi / 4$. If instead we let $\kappa=2-\epsilon, \epsilon>0$, then

$$
z_{1,2} \approx \frac{1}{2 i} \log \left(\frac{-4 \epsilon \pm(12 \epsilon)^{1 / 2}}{-\epsilon}\right)=\frac{1}{2 i} \log \left(4 \mp 2(3 / \epsilon)^{1 / 2}\right),
$$

so this time in the limit $\epsilon \rightarrow 0$ the real parts of $z_{1,2}$ approach 0 and $\pi / 2$ (see Fig. 9). At the critical level $\kappa=2$ we have the trivial equilibrium with vortices of circulation -2 at 0 corresponding to $\psi=\sin z / \sin ^{3} z=\sin ^{-2} z$ with both vortices with circulation 1 gone to infinity.

When $\kappa \rightarrow \infty$, then $X_{1,2} \rightarrow 1$ so that red vortices $z_{1,2} \rightarrow n \pi, n \in \mathbb{Z}$ approach blue one (see Fig. 10). For $\kappa<0$ the configuration will look the same as the corresponding positive configuration but will move to the left, instead.

\subsection{Case $n=2$ with $k_{1}=m, k_{2}=n, \phi_{1}=\phi_{2}=0$}

The corresponding configuration of vortices $\Sigma_{m, n, \kappa}$ is given by the zeros of

$$
\begin{aligned}
W_{m, n}= & W(\sin m z, \sin n z)=((n-m) \sin (m+n) z-(m+n) \sin (n-m) z) / 2, \\
W_{m, n}^{\kappa}= & W\left(\sin m z, \sin n z, e^{i \kappa z}\right) \\
= & \left((n+m)\left(\kappa^{2}-m n\right) \sin (n-m) z-(n-m)\left(\kappa^{2}+m n\right) \sin (n+m) z\right. \\
& \left.+i \kappa\left(n^{2}-m^{2}\right)(\cos (n-m) z-\cos (n+m) z)\right) e^{i \kappa z} / 2 .
\end{aligned}
$$

When $\kappa=0$ we have a genuine equilibrium. In that case $W_{m, n}^{0}=m n W(\cos m z, \cos n z)$, where

$$
W(\cos m z, \cos n z)=\frac{n-m}{2} \sin (n+m) z+\frac{n+m}{2} \sin (n-m) z .
$$


The number of common zeros of $W_{m, n}^{0}$ and $W_{m, n}$ depends on arithmetic of $m$ and $n$. If $d$ is the greatest common divisor of $m$ and $n$ then $W_{m, n}^{0}$ and $W_{m, n}$ have $d$ common zeros at $z=l \pi / d$, $l=0, \ldots, d-1$ of multiplicities 1 and 3 respectively. Thus we have per period $m+n-3 d$ vortices of circulation $-1, d$ vortices of circulation -2 and $m+n-d$ vortices of circulation 1 (see the example with $m=10, n=12$ below). The same is true for generic values of $\kappa$.

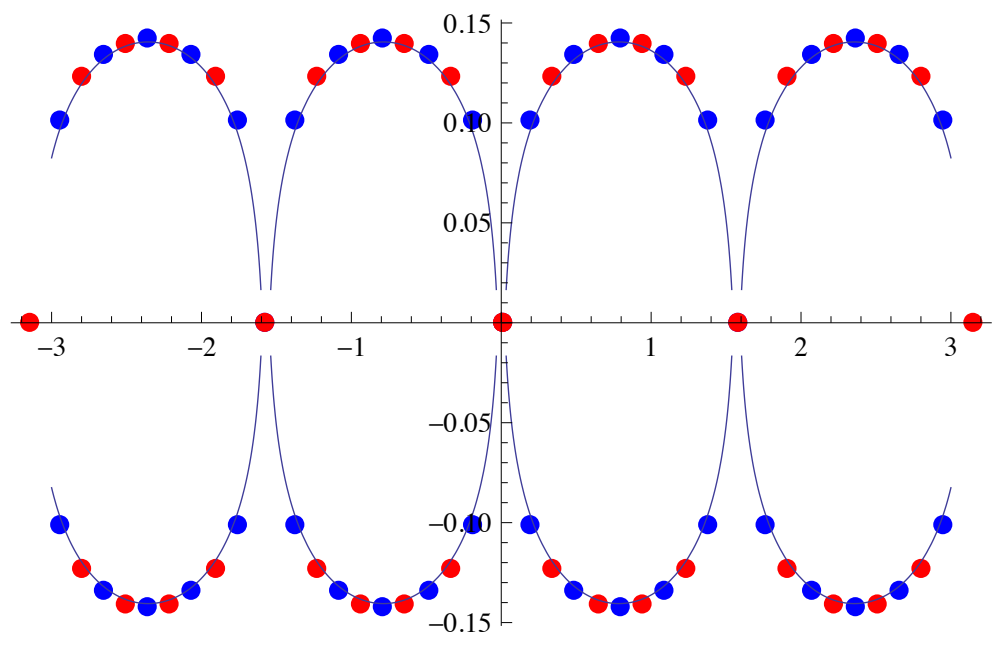

Figure 11. Vortex equilibrium configuration $\Sigma_{10,12,0,0}$ against the asymptotic curve (6.3).

Since the picture suggests that the vortices lie on some curve let us try to find its shape. Our arguments here are similar to the analysis of the Wronskians of Hermite polynomials in [12].

For complex zeros of both $W_{m, n}^{0}$ and $W_{m, n}$ we have

$$
|\sin (m+n) z|=\frac{m+n}{n-m}|\sin (n-m) z| .
$$

Let us assume that $m, n$ are large compared with the difference $m-n$. Then in the upper half-plane $z=x+i y, y>0$ the negative exponential term in $\sin (m+n) z$, with modulus $e^{(m+n) y} / 2$, will dominate. Taking the modulus of both sides and assuming that $y$ is small, so that $\sin (n-m) z \approx \sin (n-m) x$, equation (6.2) becomes

$$
\frac{1}{2} e^{(m+n) y} \approx \frac{m+n}{n-m}|\sin (n-m) x| .
$$

Taking logarithms and combining with the lower half-plane case, we have the following approximate formula for the curve on which the zeros lie

$$
|y|= \pm \frac{1}{m+n}\left(\log |\sin (n-m) x|+\log \frac{2(m+n)}{n-m}\right) .
$$

Fig. 11 shows a good agreement with this formula already for $m=7, n=8$.

When the parameter $\kappa$ (which is essentially velocity) increases from zero the red vortices lie on their own independent curves. We will now derive a formula for these curves, from equation (6.1). Setting $W_{m, n}^{\kappa}=0$, then collecting terms with argument $(n+m) z$ onto the left and those with argument $(n-m) z$ onto the right, we have

$$
\begin{aligned}
& (n-m)\left(\kappa(n+m) \cos (n+m) z-i\left(\kappa^{2}+m n\right) \sin (n+m) z\right) \\
& \quad=(n+m)\left(\kappa(n-m) \cos (n-m) z-i\left(\kappa^{2}-m n\right) \sin (n-m) z\right) .
\end{aligned}
$$


Writing the left hand side in terms of exponentials, we get

$$
\begin{aligned}
& \frac{n-m}{2}\left((\kappa-n)(\kappa-m) e^{i(n+m) z}-(\kappa+n)(\kappa+m) e^{-i(n+m) z}\right) \\
& \quad=(n+m)\left(\kappa(n-m) \cos (n-m) z-i\left(\kappa^{2}-m n\right) \sin (n-m) z\right) .
\end{aligned}
$$

Since $(n+m)$ is assumed to be large, in the upper half-plane the negative exponential term will dominate. Set $z=x+i y$ and assume that $y$ is small enough that $\sin z \approx \sin x$ and similarly for $\cos z$. Taking the modulus of both sides we have

$$
\begin{aligned}
& \frac{n-m}{2}(\kappa+n)(\kappa+m) e^{(n+m) y} \\
& \quad \approx(n+m)\left(\left(\kappa^{2}-n^{2}\right)\left(\kappa^{2}-m^{2}\right) \sin ^{2}(m-n) x+\kappa^{2}(n-m)^{2}\right)^{1 / 2} .
\end{aligned}
$$

Taking logarithms of both sides we arrive at the formula

$$
\begin{aligned}
y= & \frac{1}{2(n+m)} \log \left|\frac{\kappa-n}{\kappa+n} \frac{\kappa-m}{\kappa+m}\right|+\frac{1}{(n+m)} \log \frac{2(n+m)}{n-m} \\
& +\frac{1}{2(n+m)} \log \left|\sin ^{2}(n-m) x+\frac{\kappa^{2}(n-m)^{2}}{\left(\kappa^{2}-n^{2}\right)\left(\kappa^{2}-m^{2}\right)}\right|,
\end{aligned}
$$

and by a similar calculation in the lower half-plane (where we keep the positive exponential term instead) we have a formula for the lower line of vortices. Combining the two gives us

$$
\begin{aligned}
y_{ \pm}= & \frac{1}{2(n+m)} \log \left|\frac{\kappa-n}{\kappa+n} \frac{\kappa-m}{\kappa+m}\right| \\
& \pm \frac{1}{2(n+m)}\left(2 \log \frac{2(n+m)}{n-m}+\log \left|\sin ^{2}(n-m) x+\frac{\kappa^{2}(n-m)^{2}}{\left(\kappa^{2}-n^{2}\right)\left(\kappa^{2}-m^{2}\right)}\right|\right) .
\end{aligned}
$$

Formula (6.4) works well away from the critical values $\kappa=m$ or $\kappa=n$, when we have the equilibria corresponding to $\psi=\sin n z / W_{m, n}$ and $\psi=\sin m z / W_{m, n}$ respectively (see Figs. 13 and 14). Conjecturally the formulae (6.3), (6.4) define the asymptotic curves for the zeros in the limit of large $m$ and $n$ with fixed difference $n-m$ (cf. [12], where the Wronskians of Hermite polynomials were studied).

The configurations corresponding to $m=7$ and $n=8$ are displayed in the sequence of pictures shown in Figs. 12-15 for increasing values of the parameter $\kappa$. We give a qualitative description of what is happening at each stage:

- $\kappa=0$. The zeros of $W_{m, n}$ and $W_{m, n}^{\kappa}$ are interlaced.

- $0<\kappa<m$. The zeros of $W_{m, n}^{\kappa}$ move downward whilst maintaining a similar overall form until $\kappa$ approaches $m$, when they flatten out.

- $\kappa=m$. The first critical value. The bottom line of vortices tends to $-i \infty$. The top line of vortices sit on the real axis at the zeros of $\sin n x$.

- $m<\kappa<n$. The bottom line returns and a vortex is exchanged between the top and bottom lines.

- $\kappa=n$. The second critical case. Again, the bottom line of vortices tends to $-i \infty$ and the top line of vortices sit on the real axis, only this time at the zeros of $\sin m x$.

- $\kappa>n$. The red vortices move upwards and tend towards the blue vortices as $\kappa \rightarrow \infty$.

Recall that in all these examples the velocity is horizontal since $\kappa$ is real. 

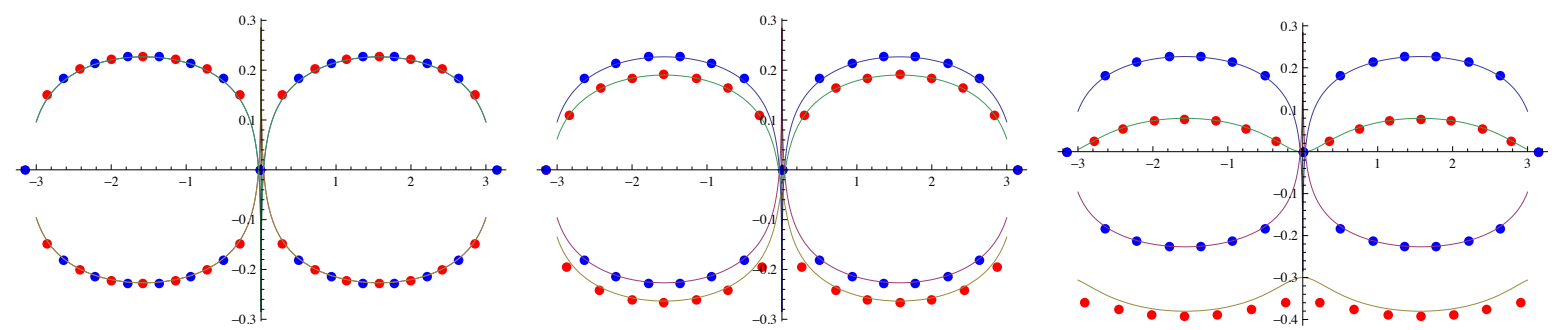

Figure 12. $\Sigma_{7,8, \kappa}$ with $\kappa=0$ (equilibrium, L), $\kappa=2(\mathrm{M}), \kappa=6(\mathrm{R})$.
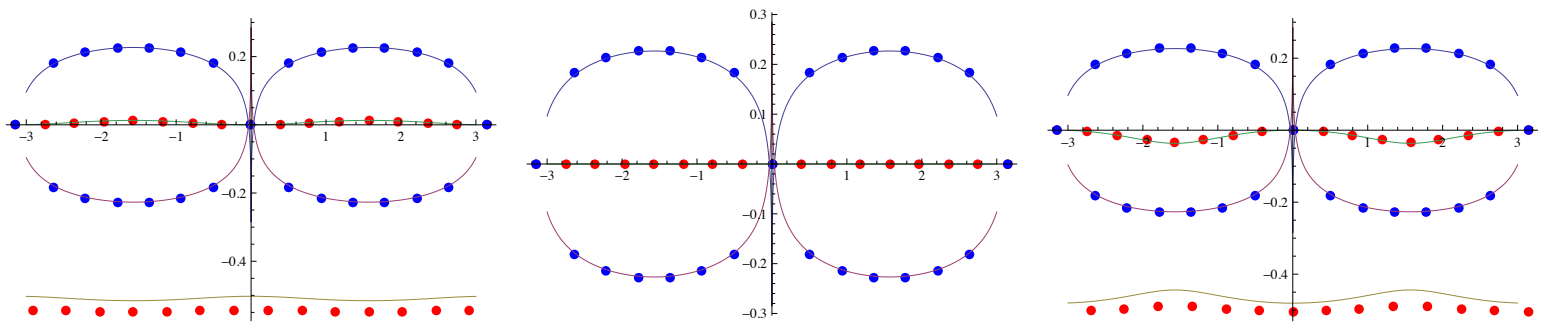

Figure 13. $\Sigma_{7,8, \kappa}$ with $\kappa=6.9(\mathrm{~L}), \kappa=7$ (critical, $\left.\mathrm{M}\right), \kappa=7.2(\mathrm{R})$.
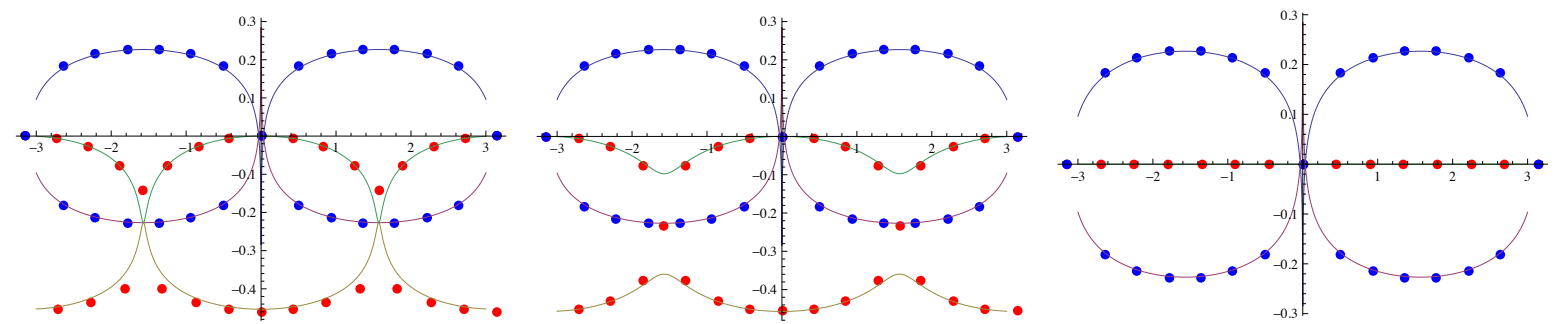

Figure 14. $\Sigma_{7,8, \kappa}$ with $\kappa=7.5(\mathrm{~L}), \kappa=7.6(\mathrm{M}), \kappa=8$ (critical, R).
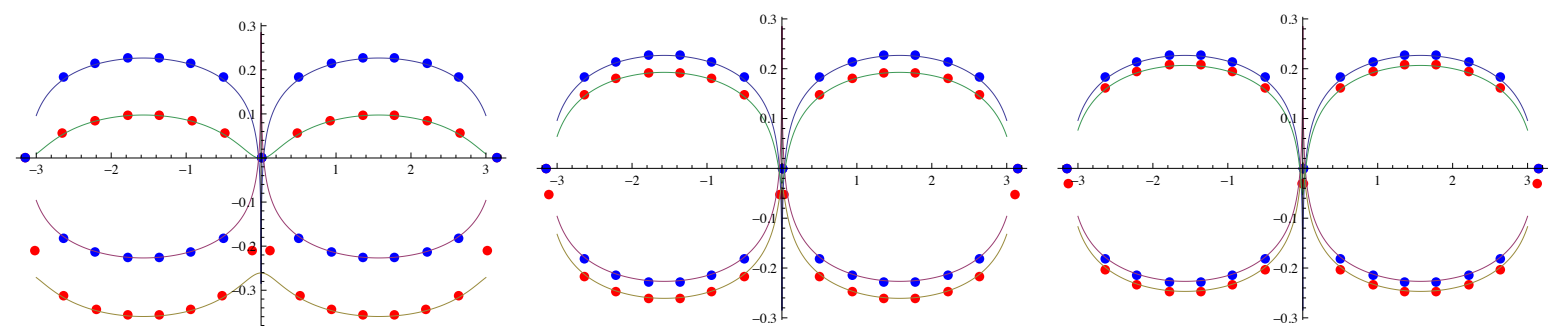

Figure 15. $\Sigma_{7,8, \kappa}$ with $\kappa=10(\mathrm{~L}), \kappa=30(\mathrm{M}), \kappa=50(\mathrm{R})$.

\section{Concluding remarks}

An explicit description of all monodromy-free operators is known only in a few cases: rational class of potentials decaying or with quadratic growth at infinity [23] and trigonometric class described in Section 3. Already in the sextic rational case the situation is far from clear, see [16] for the latest results in this direction. The same is true about monodromy-free perturbations of Whittaker-Hill operator [17] and already mentioned elliptic case.

The link with vortex dynamics adds one more reason to the importance of these problems. It would be interesting to analyse from this point of view a class of the monodromy-free potentials in terms of the Painlevé-IV transcendents described in [33]. Another interesting question is to study the quasi-periodic case by allowing in the construction of $\Sigma_{k, \phi, \kappa}$ non-integer $k_{j}$. 
The geometry of the trigonometric configurations $\Sigma_{k, \phi, \kappa}$ is also worthy of studying further, in particular, the asymptotic shape of the corresponding vortex streets. It would be nice also to see if the shape of the corresponding Young diagram of $k$ plays any role here, similarly to the case of Hermite polynomials in [12].

Finally, from the point of view of possible applications the stability of the new equilibria is crucial and is to be investigated (see Lamb [20] for the conditions of stability for the original von Kármán streets).

\section{Acknowledgements}

We are very grateful to John Gibbons and Boris Khesin for helpful and encouraging discussions. We also thank all the referees for the critical comments and constructive suggestions. This work was mainly done in spring 2012 when the first author (ADH) was a $\mathrm{PhD}$ student at Loughborough University. The work of APV was partly supported by the EPSRC (grant $\mathrm{EP} / \mathrm{J} 00488 \mathrm{X} / 1)$.

\section{References}

[1] Acheson D.J., Elementary fluid dynamics, Oxford Applied Mathematics and Computing Science Series, The Clarendon Press, Oxford University Press, New York, 1990.

[2] Airault H., McKean H.P., Moser J., Rational and elliptic solutions of the Korteweg-de Vries equation and a related many-body problem, Comm. Pure Appl. Math. 30 (1977), 95-148.

[3] Aref H., Newton P.K., Stremler M.A., Tokieda T., Vainchtein D.L., Vortex crystals, Adv. Appl. Mech. 39 (2003), 1-75.

[4] Aref H., Stremler M.A., On the motion of three point vortices in a periodic strip, J. Fluid Mech. 314 (1996), $1-25$.

[5] Bartman A.B., A new interpretation of the Adler-Moser KdV polynomials: interaction of vortices, in Nonlinear and Turbulent Processes in Physics, Vol. 3 (Kiev, 1983), Harwood Academic Publ., Chur, 1984, $1175-1181$.

[6] Berest Yu.Yu., Solution of a restricted Hadamard problem on Minkowski spaces, Comm. Pure Appl. Math. 50 (1997), 1019-1052.

[7] Berest Yu.Yu., Loutsenko I.M., Huygens' principle in Minkowski spaces and soliton solutions of the Korteweg-de Vries equation, Comm. Math. Phys. 190 (1997), 113-132, solv-int/9704012.

[8] Chalykh O.A., Feigin M.V., Veselov A.P., Multidimensional Baker-Akhiezer functions and Huygens' principle, Comm. Math. Phys. 206 (1999), 533-566, math-ph/9903019.

[9] Dubrovin B.A., Novikov S.P., Periodic and conditionally periodic analogs of the many-soliton solutions of the Korteweg-de Vries equation, Soviet Phys. JETP 40 (1975), 1058-1063.

[10] Duistermaat J.J., Grünbaum F.A., Differential equations in the spectral parameter, Comm. Math. Phys. 103 (1986), 177-240.

[11] Feigin M.V., Johnston D., A class of Baker-Akhiezer arrangements, Comm. Math. Phys. 328 (2014), 11171157, arXiv:1212.3597.

[12] Felder G., Hemery A.D., Veselov A.P., Zeros of Wronskians of Hermite polynomials and Young diagrams, Phys. D 241 (2012), 2131-2137, arXiv:1005.2695.

[13] Feynman R.P., Application of quantum mechanics to liquid helium, in Quantum Turbulence, Progress in Low Temperature Physics, Vol. 1, Editor C.J. Gorter, Amsterdam, North-Holland, 1955, 17-53.

[14] Friedman A.A., Polubarinova P.Ya., On moving singularities of planar motion of incompressible fluid, Geofiz. Sb. 5 (1927), 9-24 (in Russian).

[15] Gesztesy F., Weikard R., Picard potentials and Hill's equation on a torus, Acta Math. 176 (1996), 73-107.

[16] Gibbons J., Veselov A.P., On the rational monodromy-free potentials with sextic growth, J. Math. Phys. 50 (2009), 013513, 25 pages, arXiv:0807.3501.

[17] Hemery A.D., Veselov A.P., Whittaker-Hill equation and semifinite-gap Schrödinger operators, J. Math. Phys. 51 (2010), 072108, 17 pages, arXiv:0906.1697. 
[18] Krichever I.M., Methods of algebraic geometry in the theory of non-linear equations, Russian Math. Surveys 32 (1977), no. 6, 185-213.

[19] Krichever I.M., Elliptic solutions of the Kadomtsev-Petviashvili equation and integrable systems of particles, Funct. Anal. Appl. 14 (1980), 282-290.

[20] Lamb H., Hydrodynamics, 6th ed., Cambridge Mathematical Library, Cambridge University Press, Cambridge, 1993.

[21] Milne-Thomson L.M., Theoretical hydrodynamics, 5th ed., Macmillan, London, 1968.

[22] Montaldi J., Soulière A., Tokieda T., Vortex dynamics on a cylinder, SIAM J. Appl. Dyn. Syst. 2 (2003), 417-430, math.DS/0210028.

[23] Oblomkov A.A., Monodromy-free Schrödinger operators with quadratically increasing potential, Theoret. and Math. Phys. 121 (1999), 1574-1584.

[24] Smirnov A.O., Finite-gap solutions of the Fuchsian equations, Lett. Math. Phys. 76 (2006), 297-316, math.CA/0310465.

[25] Stieltjes T.J., Sur quelques théorèmes d'algèbre, C. R. Math. Acad. Sci. Paris 100 (1885), 439-440.

[26] Stremler M.A., On relative equilibria and integrable dynamics of point vortices in periodic domains, Theor. Comput. Fluid Dyn. 24 (2010), 25-37.

[27] Stremler M.A., Aref H., Motion of three point vortices in a periodic parallelogram, J. Fluid Mech. 392 (1999), 101-128.

[28] Taŭmanov I.A., On the two-gap elliptic potentials, Acta Appl. Math. 36 (1994), 119-124.

[29] Takemura K., The Heun equation and the Calogero-Moser-Sutherland system. I. The Bethe Ansatz method, Comm. Math. Phys. 235 (2003), 467-494, math.CA/0103077.

[30] Tkachenko V.K., On vortex lattices, Soviet Phys. JETP 22 (1966), 1282-1286.

[31] Tokieda T., Tourbillons dansants, C. R. Acad. Sci. Paris Sér. I Math. 333 (2001), 943-946.

[32] Treibich A., Verdier J.-L., Solitons elliptiques, in The Grothendieck Festschrift, Vol. III, Progr. Math., Vol. 88, Birkhäuser Boston, Boston, MA, 1990, 437-480.

[33] Veselov A.P., On Stieltjes relations, Painlevé-IV hierarchy and complex monodromy, J. Phys. A: Math. Gen. 34 (2001), 3511-3519, math-ph/0012040.

[34] Veselov A.P., On Darboux-Treibich-Verdier potentials, Lett. Math. Phys. 96 (2011), 209-216, arXiv:1004.5355.

[35] von Helmholtz H., Über Integrale der hydrodynamischen Gleichungen, welche den Wirbelbewegungen entsprechen, J. Reine Angew. Math. 55 (1858), 25-55 (English transl.: On integrals of the hydrodynamic equations which express vortex motions, Philos. Mag. 33 (1867), 485-512).

[36] von Kármán Th., Über den Mechanismus des Widerstandes, den ein bewegter Körper in einer Flüssigkeit erfährt, Nachr. Ges. Wiss. Göttingen Math.-Phys. Kl. (1911), 509-517.

[37] von Kármán Th., Über den Mechanismus des Widerstandes, den ein bewegter Körper in einer Flüssigkeit erfährt, Nachr. Ges. Wiss. Göttingen Math.-Phys. Kl. (1912), 547-556.

[38] Whittaker E.T., On a class of differential equations whose solutions satisfy integral equations, Proc. Edinburgh Math. Soc. 33 (1914), 14-23.

[39] Whittaker E.T., Watson G.N., A course of modern analysis, Cambridge Mathematical Library, Cambridge University Press, Cambridge, 1996. 